\title{
Short Communication/Kurze Mitteilung A Fast Algorithm for Solving Special Tridiagonal Systems
}

\author{
W.-M. Yan and K.-L. Chung, Taipei
}

Received June 18, 1993; revised November 11, 1993

\begin{abstract}
Zusammenfassung
A Fast Algorithm for Solving Special Tridiagonal Systems. In this paper, a fast algorithm for solving the special tridiagonal system is presented. This special tridiagonal system is a symmetric diagonally dominant and Toeplitz system of linear equations. The error analysis is also given. Our algorithm is quite competitive with the Gaussian elimination, cyclic reduction, special $L U$ factorization, reversed triangular factorization, and Toeplitz factorization methods. In addition, our result can be applied to solve the near-Toeplitz tridiagonal system. Some examples demonstrate the good efficiency and stability of our algorithm.
\end{abstract}

AMS Subject Classification: $65 \mathrm{~F}$

Key words: Diagonally dominant matrices, error analysis, linear recurrences, Toeplitz matrices, tridiagonal matrices.

Ein schneller Algorithmus zur Lösung spezieller tridiagonaler Systeme. In dieser Arbeit wird ein schneller Algorithmus zur Lösung symmetrischer, diagonaldominanter tridiagonaler Töpflitz-Systeme vorgestellt. Auch eine Fehleranalyse liegt vor. Der Algorithmus ist den folgenden Verfahren mindestens gleichwertig: Gauss-Elimination, zyklische Reduktion, spezielle $L U$-Faktorisierung, umgekehrte Faktorisierung, Töplitz-Faktorisierung. Außerdem kann unser Vorgehen zur Lösung in tridiagonalen fast-TöplitzSystemen verwendet werden. Einige Beispiele zeigen die Effizienz und Stabilität unseres Algorithmus.

\section{Introduction}

Consider an $n \times n$ system of linear equations

$$
A \mathbf{x}=\mathbf{b} \text {, }
$$

where

$$
A=\left(\begin{array}{llllll}
\beta & \gamma & & & & \gamma \\
\gamma & \beta & \gamma & & & \\
& \cdot & \cdot & \cdot & & \\
& & & \gamma & \beta & \gamma \\
\gamma & & & & \gamma & \beta
\end{array}\right)
$$

and $|\beta|>2|\gamma|$. That is, the matrix $A$ is strictly diagonally dominant. Special tridiagonal systems of equations having symmetric circulant coefficient matrices 
appear in many applications ([8], [9], [11], [14], [13]). Many methods have been proposed for solving such systems. These methods are Gaussian elimination [4], cyclic reduction [9], special $L U$ factorization ([14], [10]), reversed triangular factorization ([5], [6], [7]), and Toeplitz factorization with Sherman-Morrison formula ([8], [12], [3]). The interested readers are suggested to consult the survey paper by Boisvert [2].

In this paper, a fast algorithm for solving the special tridiagonal system is presented. Our algorithm consists of three phases. The first phase is a Toeplitz factorization of a slightly perturbed system, which is similar to the method of Fischer et al. [8]. The second phase uses a forward and backward substitution procedure to solve the perturbed problem. In the third phase the solution to the original problem is recovered from the solution to the perturbed problem; this is called the update procedure. The major contributions of our algorithm are twofold: (1) based on the diagonally dominant property, a new but simple update procedure is proposed and (2) a detailed error analysis is given. Our efficient and stable algorithm is quite competitive with previous methods [2]. In addition, our result can be applied to solve near-Toeplitz tridiagonal systems. It also can be applied to solve the quadratic B-spline curve fitting problem [11] and a parabolic PDE [13].

We begin by presenting our algorithm for solving the symmetric Toeplitz tridiagonal system. We then give an extension to the symmetric circulant tridiagonal case.

\section{Symmetric Toeplitz Tridiagonal Systems}

Throughout this paper, matrices are represented by uppercase letters, vectors by bold lowercase letters, and scalars by plain lowercase letters. The superscripts $T$ correspond to the transpose operation.

We first consider to solve the symmetric Toeplitz tridiagonal system $B \mathbf{x}=\mathbf{b}$, where

$$
B=\left(\begin{array}{llllll}
\beta & \gamma & & & & \\
\gamma & \beta & \gamma & & & \\
& \cdot & \cdot & \cdot & & \\
& & & \gamma & \beta & \gamma \\
& & & \gamma & \beta
\end{array}\right),
$$

and $|\beta|>2|\gamma|$. Let $d=\frac{\beta}{\gamma}(|d|>2)$ and

$$
B^{\prime}=\left[\begin{array}{llllll}
a & 1 & & & & \\
1 & d & 1 & & & \\
& \cdot & \cdot & \cdot & & \\
& & & 1 & d & 1 \\
& & & & 1 & d
\end{array}\right]=L U
$$


where

$$
L=\left[\begin{array}{cccccc}
1 & & & & & \\
-b & 1 & & & & \\
& \cdot & \cdot & & & \\
& & & -b & 1 & \\
& & & & -b & 1
\end{array}\right] \text { and } U=\left(\begin{array}{cccccc}
a & 1 & & & & \\
& a & 1 & & & \\
& & \cdot & . & & \\
& & & a & 1 \\
& & & & a
\end{array}\right],
$$

which implies that $a-b=d$ and $-a b=1$. This, in turn implies that $a=$ $\left(d \pm \sqrt{d^{2}-4}\right) / 2$. Since we wish the matrix $U$ to be diagonally dominant, we will select the sign so that the absolute value of $a$ is greater than 1 . That is, when $d>2$, we chose $a=\left(d+\sqrt{d^{2}-4}\right) / 2(>d / 2>1)$, from which

$$
b=a-d=\left(-d+\sqrt{d^{2}-4}\right) / 2 .
$$

When $d<-2$, we chose $a=\left(d-\sqrt{d^{2}-4}\right) / 2(<d / 2<-1)$, then

$$
b=a-d=\left(-d-\sqrt{d^{2}-4}\right) / 2 .
$$

Since $|a b|=1$ and $|a|>1$, we have that $|b|<1$, that is, the matrix $L$ is diagonally dominant. The computation of $a$ and $b$ provides the Toeplitz factorization of the matrix $B^{\prime}$, which can be done in $O(1)$ time.

By (2), $d=\frac{\beta}{\gamma}$, and $a-b=d$, it is clear that

$$
B=\gamma B^{\prime}+\left[\begin{array}{c}
-\gamma b \\
\end{array}\right]
$$

To solve $B \mathbf{x}=\mathbf{b}$, we first solve $B^{\prime} \mathbf{x}^{\prime}=\frac{1}{\gamma} \mathbf{b}\left(=\mathbf{b}^{\prime}\right)$ using a forward and backward substitution procedure (second phase in our algorithm). It is not hard to verify that the number of floating-point operations required in this procedure is about $5 n$.

By (3) and $\mathbf{b}=\gamma \mathbf{b}^{\prime}$, we obtain

$$
\begin{aligned}
B \mathbf{x}^{\prime} & =\gamma B^{\prime} \mathbf{x}^{\prime}-\gamma b x_{1}^{\prime} \mathbf{e}_{1} \\
& =\mathbf{b}-\gamma b x_{1}^{\prime} \mathbf{e}_{1},
\end{aligned}
$$

where $\mathbf{e}_{1}=(1,0, \ldots, 0)^{t}$, Note that (4) can be derived from Sherman-Morrison formula [1] and the equality $B^{\prime-1} \mathbf{e}_{1}=\gamma\left(1-b \mathbf{e}_{1}^{t} B^{\prime-1} \mathbf{e}_{1}\right) B^{-1} \mathbf{e}_{1}$ which can be derived with some effort. Therefore, the solution $\mathbf{x}$ to be determined is $\mathbf{x}^{\prime}+\gamma b x_{1}^{\prime} B^{-1} \mathbf{e}_{1}$. If we can find a vector $\mathbf{p}$ such that $B \mathbf{p}$ is equal to a multiple of $\mathbf{e}_{1}$, then we can solve $B \mathbf{x}=\mathbf{b}$ approximately by adding a multiple of $\mathbf{p}$ to $\mathbf{x}^{\prime}$. 
To find $\mathbf{p}$, we first solve the recurrence relation: $\gamma p_{i-1}+\beta p_{i}+\gamma p_{i+1}=0$ for $2 \leq i \leq$ $n-1$. From $a-b=d,-a b=1$, and $d=\frac{\beta}{\gamma}$, it follows $b^{2}+d b+1=0$ and $\gamma b^{2}+$ $\beta b+\gamma=0$. Hence, $b$ is a zero of the characteristic polynomial of the above recurrence relation. Naturally, if we try $\mathbf{p}=\left(b, b^{2}, \ldots, b^{t}, 0, \ldots, 0\right)^{T}$, then

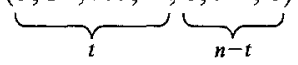

$$
\begin{aligned}
\frac{1}{\gamma} B \mathbf{p} & =(\underbrace{d b+b^{2}, b+d b^{2}+b^{3}, \ldots, b^{t-1}+d b^{t}}_{t} \underbrace{b^{t}, 0, \ldots, 0}_{n-t})^{T} \\
& =\underbrace{\left(d b+b^{2}, 0, \ldots, 0, b^{t-1}+d b^{t}\right.}_{t}, \underbrace{b^{t}, 0, \ldots, 0}_{n-t})^{T} \\
& =-\mathbf{e}_{1}-b^{t+1} \mathbf{e}_{t}+b^{t} \mathbf{e}_{t+1} \\
& =-\mathbf{e}_{1}+b^{t}\left(\mathbf{e}_{t+1}-b \mathbf{e}_{t}\right) .
\end{aligned}
$$

If

$$
\mathbf{x}=\mathbf{x}^{\prime}-b x_{1}^{\prime} \mathbf{p}
$$

then by (6), (4), and (5), we obtain $B \mathbf{x}-\mathbf{b}=-b^{t+1} \gamma x_{1}^{\prime}\left(\mathbf{e}_{t+1}-b \mathbf{e}_{t}\right)$. The update procedure in (6) is the third phase in our algorithm. It is not hard to verify that the number of floating-point operations required in the update procedure (6) is about $2 t$. Taking sup-norm on both sides, because of $|b|<1$, it gives

$$
\|B \mathbf{x}-\mathbf{b}\|=\left|b^{t+1} \gamma x_{1}^{\prime}\right| \leq \mid b^{t+1} \gamma\left\|\mathbf{x}^{\prime}\right\| .
$$

To estimate $\left\|\mathbf{x}^{\prime}\right\|$, we need the following lemma.

Lemma 1. If $B^{\prime} \mathbf{x}^{\prime}=\mathbf{b}^{\prime}$, then $\left\|\mathbf{x}^{\prime}\right\| \leq \frac{1}{|d|-2}\left\|\mathbf{b}^{\prime}\right\|$.

Proof: Because of $B^{\prime} \mathbf{x}^{\prime}=\mathbf{b}^{\prime}$, suppose $\left\|\mathbf{x}^{\prime}\right\|=\left|x_{i}^{\prime}\right|$ for some $i, 2 \leq i \leq n-1$, we have

$$
d x_{i}^{\prime}=b_{i}^{\prime}-x_{i-1}^{\prime}-x_{i+1}^{\prime} .
$$

Using the triangle inequality, it follows that

$$
\begin{aligned}
\left\|d \mathbf{x}^{\prime}\right\| & =\left|d x_{i}^{\prime}\right| \\
& \leq\left|b_{i}^{\prime}\right|+\left|x_{i-1}^{\prime}\right|+\left|x_{i+1}^{\prime}\right| \\
& \leq\left\|\mathbf{b}^{\prime}\right\|+2\left\|\mathbf{x}^{\prime}\right\| \\
\left\|\mathbf{x}^{\prime}\right\| & \leq \frac{1}{|d|-2}\left\|\mathbf{b}^{\prime}\right\| .
\end{aligned}
$$

The cases $\left\|\mathbf{x}^{\prime}\right\|=\left|x_{1}^{\prime}\right|$ and $\left\|\mathbf{x}^{\prime}\right\|=\left|x_{n}^{\prime}\right|$ are considered next. Suppose $\left\|\mathbf{x}^{\prime}\right\|=\left|x_{1}^{\prime}\right|$, we have

$$
a x_{1}^{\prime}=b_{1}^{\prime}-x_{2}^{\prime}
$$


Using the triangle inequality again, it follows that

$$
\begin{aligned}
\left\|a \mathbf{x}^{\prime}\right\| & =\left|a x_{1}^{\prime}\right| \\
& \leq\left|b_{1}^{\prime}\right|+\left|x_{2}^{\prime}\right| \\
& \leq\left\|\mathbf{b}^{\prime}\right\|+\left\|\mathbf{x}^{\prime}\right\| \\
\left\|\mathbf{x}^{\prime}\right\| & \leq \frac{1}{|a|-1}\left\|\mathbf{b}^{\prime}\right\| .
\end{aligned}
$$

Finally, suppose $\left\|\mathbf{x}^{\prime}\right\|=\left|x_{n}^{\prime}\right|$, we have

$$
d x_{n}^{\prime}=b_{n}^{\prime}-x_{n-1}^{\prime} .
$$

Similarly, it follows that

$$
\begin{aligned}
\left\|d \mathbf{x}^{\prime}\right\| & =\left|d x_{n}^{\prime}\right| \\
& \leq\left|b_{n}^{\prime}\right|+\left|x_{n-1}^{\prime}\right| \\
& \leq\left\|\mathbf{b}^{\prime}\right\|+\left\|\mathbf{x}^{\prime}\right\| \\
\left\|\mathbf{x}^{\prime}\right\| & \leq \frac{1}{|d|-1}\left\|\mathbf{b}^{\prime}\right\| .
\end{aligned}
$$

We conclude that $\left\|\mathbf{x}^{\prime}\right\| \leq \max \left(\frac{1}{|d|-2}, \frac{1}{|a|-1}, \frac{1}{|d|-1}\right)\left\|\mathbf{b}^{\prime}\right\|$. Since $a-b=d$ and $|b|<1$, we have $|d| \leq|a|+|b|<|a|+1$, and hence $0<|d|-2<|a|-1$, because of $|d|>2$. Hence, we obtain $\max \left(\frac{1}{|d|-2}, \frac{1}{|a|-1}, \frac{1}{|d|-1}\right)=\frac{1}{|d|-2}$. Therefore, we have $\left\|\mathbf{x}^{\prime}\right\| \leq \frac{1}{|d|-2}\left\|\mathbf{b}^{\prime}\right\|$.

By (7), Lemma 1, and $\mathbf{b}=\gamma \mathbf{b}^{\prime}$, we have

$$
\begin{aligned}
\|B \mathbf{x}-\mathbf{b}\| & \leq\left|b^{t+1} \gamma\right|\left\|\mathbf{x}^{\prime}\right\| \\
& \leq\left|b^{t+1} \gamma\right| \frac{\left\|\mathbf{b}^{\prime}\right\|}{|d|-2} \\
& =\frac{\left|b^{t+1}\right|\|\mathbf{b}\|}{|d|-2} .
\end{aligned}
$$

Immediately, we have the following theorem.

Theorem 2. $\|B \mathbf{x}-\mathbf{b}\| \leq \frac{\left|b^{t+1}\right|\|\mathbf{b}\|}{|d|-2}$.

Recall that $|b|<1$, so the bound of $\|B x-b\|$ is decayed exponentially in terms of $b$. For example, if we hope $\|B \mathbf{x}-\mathbf{b}\|<\xi\|\mathbf{b}\|$, then

$$
t(\xi) \geq \frac{\log (|d|-2)+\log \xi}{\log |b|}-1 .
$$


The smallest $t(\xi)$ of (8) will be small enough for some relative tolerance $\xi$ and sufficiently large diagonal dominance ratio $|d|$. Using (8), Table 1 illustrates some relations between $|d|$ 's and the smallest $t$ 's for some $\xi$. Note that the tolerance $\xi$ cannot be achieved when $t(\xi)>n$, and hence the algorithm will break down when the required relative tolerance is sufficiently small and/or the diagonal dominance ratio is sufficiently close to 2 .

Table 1

\begin{tabular}{|c|c|c|c|c|}
\hline$|d|$ & $t\left(10^{-2}\right)$ & $t\left(10^{-4}\right)$ & $t\left(10^{-6}\right)$ & $t\left(10^{-8}\right)$ \\
\hline 2.001 & 364 & 509 & 655 & 800 \\
2.01 & 92 & 138 & 184 & 230 \\
2.05 & 34 & 54 & 75 & 95 \\
2.1 & 21 & 36 & 51 & 65 \\
2.5 & 7 & 14 & 20 & 27 \\
4 & 2 & 6 & 9 & 13 \\
6 & 1 & 4 & 7 & 9 \\
8 & 1 & 3 & 5 & 8 \\
\hline
\end{tabular}

In summary, based on our three-phase algorithm described in this section, solving the symmetric Toeplitz tridiagonal system $B \mathbf{x}=\mathbf{b}$ takes about $5 n+2 t$. For some relative tolerance and highly diagonal dominance ratio, we have that $t$ is $O(1)$. That is, it takes about $5 n$ to solve $B \mathbf{x}=\mathbf{b}$ for this case. The number of floating point operations required in our algorithm is the same as the previous fastest ones such as the special $L U$ factorization and reversed triangular factorization [2].

\section{Symmetric Circulant Tridiagonal Systems}

We now consider the symmetric circulant tridiagonal system $A \mathbf{x}=\mathbf{b}$, where

$$
A=\left[\begin{array}{llllll}
\beta & \gamma & & & & \gamma \\
\gamma & \beta & \gamma & & & \\
& \cdot & \cdot & \cdot & & \\
& & & \gamma & \beta & \gamma \\
\gamma & & & & \gamma & \beta
\end{array}\right] .
$$

By (2), $d=\frac{\beta}{\gamma}$, and $a-b=d$, we have

$$
A=\gamma B^{\prime}+\left[\begin{array}{cc}
-\gamma b & \gamma \\
\gamma &
\end{array}\right]
$$



To solve $A \mathbf{x}=\mathbf{b}$, we first solve $B^{\prime} \mathbf{x}^{\prime}=\frac{1}{\gamma} \mathbf{b}\left(=\mathbf{b}^{\prime}\right)$ as described in Section 2. From
(10), we obtain

$$
\begin{aligned}
A \mathbf{x}^{\prime} & =\gamma B^{\prime} \mathbf{x}^{\prime}+\gamma\left(x_{n}^{\prime}-b x_{1}^{\prime}\right) \mathbf{e}_{1}+\gamma x_{1}^{\prime} \mathbf{e}_{n} \\
& =\mathbf{b}+\gamma\left(x_{n}^{\prime}-b x_{1}^{\prime}\right) \mathbf{e}_{1}+\gamma x_{1}^{\prime} \mathbf{e}_{n} .
\end{aligned}
$$

From $b^{2}+d b+1=0$ (derived in Section 2), it follows that

$$
\begin{aligned}
\frac{1}{\gamma} A \mathbf{p} & =\underbrace{(\underbrace{d b+b^{2}, b+d b^{2}+b^{3}, \ldots, b^{t-1}+d b^{t}} \underbrace{b^{t}, 0, \ldots, 0, b}_{n-t})^{T}}_{t} \\
& =\underbrace{\left(d b+b^{2}, 0, \ldots, 0, b^{t-1}+d b^{t}\right.}_{t}, \underbrace{b^{t}, 0, \ldots, 0, b}_{n-t})^{T} \\
& =-\mathbf{e}_{1}-b^{t+1} \mathbf{e}_{t}+b^{t} \mathbf{e}_{t+1}+b \mathbf{e}_{n} \\
& =\left(-\mathbf{e}_{1}+b \mathbf{e}_{n}\right)+b^{t}\left(\mathbf{e}_{t+1}-b \mathbf{e}_{t}\right) .
\end{aligned}
$$

With respect to the reverse form of $\mathbf{p}$, naturally, we let $\mathbf{q}=(\underbrace{0, \ldots, 0}_{n-t}, \underbrace{b^{t}, \ldots, b^{2}, b^{1}})^{T}$, from which

$$
\begin{aligned}
\frac{1}{\gamma} A \mathbf{q} & =\left(-\mathbf{e}_{n}+b \mathbf{e}_{1}\right)+b^{t}\left(\mathbf{e}_{n-t}-b \mathbf{e}_{n-t+1}\right) \\
& =\left(b \mathbf{e}_{1}-\mathbf{e}_{n}\right)+b^{t}\left(\mathbf{e}_{n-t}-b \mathbf{e}_{n-t+1}\right) .
\end{aligned}
$$

Let $\mathbf{u}=\frac{1}{\gamma}(\mathbf{p}+b \mathbf{q})$ and $\mathbf{v}=\frac{1}{\gamma}(b \mathbf{p}+\mathbf{q})$. By (12) and (13), we have

$$
\begin{aligned}
& A \mathbf{u}=\left(b^{2}-1\right) \mathbf{e}_{1}+b^{t}\left(\mathbf{e}_{t+1}-b \mathbf{e}_{t}+b \mathbf{e}_{n-t}-b^{2} \mathbf{e}_{n-t+1}\right) \\
& A \mathbf{v}=\left(b^{2}-1\right) \mathbf{e}_{n}+b^{t}\left(b \mathbf{e}_{t+1}-b^{2} \mathbf{e}_{t}+\mathbf{e}_{n-t}-b \mathbf{e}_{n-t+1}\right) .
\end{aligned}
$$

Let

$$
\mathbf{x}=\mathbf{x}^{\prime}-\frac{\gamma}{b^{2}-1}\left[\left(x_{n}^{\prime}-b x_{1}^{\prime}\right) \mathbf{u}+x_{1}^{\prime} \mathbf{v}\right]
$$

by (15), (11), and (14), we obtain

$$
\begin{aligned}
A \mathbf{x}-\mathbf{b}= & -\frac{\gamma b^{t}}{b^{2}-1}\left[x_{n}^{\prime} \mathbf{e}_{t+1}-b x_{n}^{\prime} \mathbf{e}_{t}+\left(b x_{n}^{\prime}+\left(1-b^{2}\right) x_{1}^{\prime}\right) \mathbf{e}_{n-t}\right. \\
& \left.-\left(b^{2} x_{n}^{\prime}+\left(b-b^{3}\right) x_{1}^{\prime}\right) \mathbf{e}_{n-t+1}\right] .
\end{aligned}
$$

Suppose $\{t+1, t\} \cap\{n-t, n-t+1\}=\varnothing$. Since $|b|<1$, we have

$$
\begin{aligned}
\|A \mathbf{x}-\mathbf{b}\| & =\left|\frac{\gamma b^{t}}{b^{2}-1}\right| \max \left(\left|x_{n}^{\prime}\right|,\left|b x_{n}^{\prime}+\left(1-b^{2}\right) x_{1}^{\prime}\right|\right) \\
& \leq\left|\frac{\gamma b^{t}}{b^{2}-1}\right|\left(|b|+\left|1-b^{2}\right|\right)\left\|\mathbf{x}^{\prime}\right\| .
\end{aligned}
$$


Using the same arguments as Lemma 1, we also have $\left\|\mathbf{x}^{\prime}\right\| \leq \frac{1}{|d|-2}\left\|\mathbf{b}^{\prime}\right\|$. Therefore,

$$
\begin{aligned}
\|A \mathbf{x}-\mathbf{b}\| & \leq\left|\frac{\gamma b^{t}}{b^{2}-1}\right|\left(|b|+\left|1-b^{2}\right|\right) \frac{\left\|\mathbf{b}^{\prime}\right\|}{|d|-2} \\
& =\frac{\left(|b|+\left|1-b^{2}\right|\right)\left|b^{t}\right|}{\left|b^{2}-1\right|(|d|-2)}\|\mathbf{b}\| .
\end{aligned}
$$

So, the bound of $\|A \mathbf{x}-\mathbf{b}\|$ is decayed exponentially in terms of $b$. For example, if we require $\|A \mathbf{x}-\mathbf{b}\|<\xi\|\mathbf{b}\|$, then

$$
t(\xi) \geq \frac{\log (|d|-2)+\log \left|b^{2}-1\right|-\log \left(|b|+\left|1-b^{2}\right|\right)+\log \xi}{\log |b|}-1 .
$$

Table 2 illustrates some relations between $|d|$ 's and the smallest $t$ 's for some $\xi$. Note that the tolerance $\xi$ cannot be achieved when $t(\xi)>n$, and hence the algorithm will break down when the required relative tolerance is sufficiently small and/or the diagonal dominance ratio is sufficiently close to 2 .

Table 2

\begin{tabular}{|c|c|c|c|c|}
\hline$|d|$ & $t\left(10^{-2}\right)$ & $t\left(10^{-4}\right)$ & $t\left(10^{-6}\right)$ & $t\left(10^{-8}\right)$ \\
\hline 2.001 & 454 & 599 & 745 & 891 \\
2.01 & 111 & 157 & 203 & 249 \\
2.05 & 40 & 60 & 81 & 102 \\
2.1 & 25 & 40 & 55 & 69 \\
2.5 & 9 & 16 & 22 & 29 \\
4 & 4 & 7 & 11 & 14 \\
6 & 2 & 5 & 8 & 10 \\
8 & 2 & 4 & 6 & 9 \\
\hline
\end{tabular}

By (15), we have

$$
\begin{aligned}
\mathbf{x} & =\mathbf{x}^{\prime}-\frac{\gamma}{b^{2}-1}\left(\left(x_{n}^{\prime}-b x_{1}^{\prime}\right) \mathbf{u}+x_{1}^{\prime} \mathbf{v}\right) \\
& =\mathbf{x}^{\prime}-\frac{1}{b^{2}-1}\left(\left(x_{n}^{\prime}-b x_{1}^{\prime}\right)(\mathbf{p}+b \mathbf{q})+x_{1}^{\prime}(b \mathbf{p}+\mathbf{q})\right) \\
& =\mathbf{x}^{\prime}-\frac{1}{b^{2}-1}\left(x_{n}^{\prime} \mathbf{p}+\left(b x_{n}^{\prime}+\left(1-b^{2}\right) x_{1}^{\prime}\right) \mathbf{q}\right) \\
& =\mathbf{x}^{\prime}-\frac{x_{n}^{\prime}}{b^{2}-1} \mathbf{p}+\left(x_{1}^{\prime}-\frac{b x_{1}^{\prime}}{b^{2}-1}\right) \mathbf{q} .
\end{aligned}
$$

It is easy to verify that the number of floating-point operations required in the above update procedure is about $4 t$. In summary, based on our three-phase algorithm, solving the symmetric circulant Toeplitz tridiagonal system $A \mathbf{x}=\mathbf{b}$ takes about $5 n+4 t$. For some relative tolerance and highly diagonal dominance ratio, we have 
that $t$ is $O(1)$ too. It follows that the number of floating-point operations needed in our algorithm can complete with the previous fastest ones [2]. Following the derivations of our three-phase algorithm, the special near-Toeplitz tridiagonal system $C \mathbf{x}=\mathbf{b}$ can be solved in a similar way, where

$$
C=\left[\begin{array}{cccccc}
\alpha & \gamma & & & & \gamma \\
\gamma & \beta & \gamma & & & \\
& \cdot & \cdot & \cdot & & \\
& & & \gamma & \beta & \gamma \\
\gamma & & & & \gamma & \alpha^{\prime}
\end{array}\right]
$$

and $C$ is diagonally dominant.

\section{Acknowledgements}

The authors are indebted to the referee for several insightful comments. These comments improved the quality and presentation of this paper.

\section{References}

[1] Bartlett, M. S.: An inverse matrix adjustment arising in discriminant analysis. Ann. Math. Statist. 22, 107-111(1951).

[2] Boisvert, R. F.: Algorithms for special tridiagonal systems. SIAM J. Sci. Stat. Comput. 12, 423-442 (1991).

[3] Chen, M. K.: On the solution of circulant linear systems. SIAM J. Numer. Anal. 24, 668-683 (1987).

[4] Dongarra, J. J., Moler, C. B., Bunch, J. R., Stewart, G. W.: LINPACK user's guide. SIAM Press, 1979.

[5] Evans, D. J., Forrington, C. V. D.: Note on the solution of certain tri-diagonal systems of linear equations. Comput. J. 5, 327-328 (1963).

[6] Evans, D. J.: An algorithm for the solution of certain tri-diagonal systems of linear equations. Comput. J. 15, 356-359 (1972).

[7] Evans, D. J.: On the solution of certain Toeplitz tridiagonal linear systems. SIAM J. Numer. Anal. $17,675-680(1980)$.

[8] Fischer, D., Golub, G., Hald, O., Levia, C., Winlund, O.: On Fourier-Toeplitz methods for separable elliptic problems. Math. Comput. 28, 349-368 (1974).

[9] Hockney, R. W.: A fast direct solution of Poisson's equation using Fourier analysis. J. ACM. 12, 95-113(1965).

[10] Malcolm, M. A., Palmer, J.: A fast method for solving a class of tridiagonal linear systems. Comm. ACM. 17, 14-17 (1974).

[11] Pham, B.: Quadratic B-splines for automatic curve and surface fitting. Comput. Graphics 13, $471-475$ (1989).

[12] Rojo, O.: A new method for solving symmetric circulant tridiagonal systems of linear equations. Comput. Math. Appl. 20,61-67 (1990).

[13] Smith, G. D.: Numerical solution of partial differential equations: finite difference methods, 3rd edn. New York: Oxford University Press 1985.

[14] Widlund, O. B.: On the use of fast methods for separable finite difference equations for the solution of general elliptic problems. In: Sparse matrices and their applications (Rose, D. J., Willoughby, R. A., eds.), pp. 121-131. New York: Plenum Press 1972.

Mr. W.-M. Yan

Department of Computer Science

and Information Engineering

National Taiwan University

Taipei, Taiwan 10764, R.O.C.

Taiwan
Dr. K.-L. Chung

Department of Information Management

National Taiwan Institut of Technology

Taipei, Taiwan 10672, R.O.C.

Taiwan 